\title{
The effect of population distribution measures on evaluating spatial accessibility of primary health-care institutions: A case study from China
}

\author{
Jianxia Tan, ${ }^{1,2}$ Xiuli Wang, ${ }^{1,2}$ Jay Pan ${ }^{1,2}$ \\ ${ }^{1}$ HEOA Group, West China School of Public Health and West China Fourth Hospital, Sichuan University; \\ ${ }^{2}$ Institue for Healthy Cities and West China Research Center for Rural Health Development, Sichuan \\ University, Chengdu, China
}

\begin{abstract}
Improvement of the equality of geographical allocation of limited health-care resources requires an accurate evaluation of spatial accessibility of the facilities. The adoption of appropriate population distribution measures is one of the leading factors affecting such an evaluation. Using primary health-care institutions in Hainan, China as an example, this study aimed to explore the disparities embedded
\end{abstract}

Correspondence: Jay Pan, West China School of Public Health and West China Fourth Hospital, Sichuan University, No.16 People's South Road, Chengdu 610041, China.

Tel.: +86.028.8550.1272 - Fax: +86.028.8550.1528.

E-mail: panjie.jay@scu.edu.cn

Key words: Spatial accessibility; enhanced two-step floating catchment area; population distribution; night-time light; primary health care; China.

Acknowledgements: The authors thank Yili Yang from Institute for Healthy Cities, Sichuan University for her excellent language proofreading.

Funding: This study was funded by the National Natural Science Foundation of China (Grant No. 71874116, 72074163 and 42071379), Ministry of Education of China (Grant No. 18YJA790062 and 20YJC790179), Chengdu Federation of Social Science Association (Grant No. ZZ05), Sichuan University (Grant No. 2018hhf-27, SKSYL201811 and 2019SCU12013), China Medical Board (Grant No. 17-276), Sichuan University Interdisciplinary Postdoctoral Innovation Fund, China Postdoctoral Science Foundation (Grant No. 2020M673274), and Sichuan Science and Technology Program (Grant No. 2020JDR0151).

Contributions: JP, conceptualization, supervision; JT, writing original draft; JT and XW, data analysis; XW and JP, funding acquisition; JT, $\mathrm{XW}$ and JP, writing, review and editing.

Conflict of interests: the authors declare no potential conflict of interests.

Received for publication: 2 September 2020.

Revision received: 21 December 2020.

Accepted for publication: 21 December 2020.

(C) Copyright: the Author(s), 2021

Licensee PAGEPress, Italy

Geospatial Health 2021; 16:936

doi:10.4081/gh.2021.936

This article is distributed under the terms of the Creative Commons Attribution Noncommercial License (CC BY-NC 4.0) which permits any noncommercial use, distribution, and reproduction in any medium, provided the original author(s) and source are credited. in spatial accessibility evaluations based on six common measures of population distribution, namely community/village population (VillagePop), average population distribution (AveragePop), population distribution by night-time light intensity (NighttimelightPop) together with the public population databases LandScan, WorldPop and PoiPop for construction of the weights. The enhanced two-step floating catchment area method, two-way analysis of variance (ANOVA), Dunnett test, root mean square error and the mean absolute error were employed to assess and compare spatial accessibilities based on these different population distribution measures. The spatial accessibility of primary health-care institutions in Hainan was found to vary when plotted using the various population distribution measures mentioned. As indicated by the statistical outcomes of both ANOVA and the Dunnett test, using the spatial accessibility calculated by VillagePop as reference, those calculated by AveragePop and PoiPop were found to be significantly different. In addition, the spatial accessibilities calculated by AveragePop and PoiPop demonstrated higher error rates in the identification of underserved areas compared with the reference. Considering the limitations of public population databases, the adoption of nighttime light data is highly recommended for estimating population distribution in the absence of high-resolution data.

\section{Introduction}

Variability in access to health care has been identified as a main cause for inequality in health outcomes (Yip et al., 2019). To better evaluate this term, access has been classified into potential access and realized access, with its specific dimensions defined as availability, accessibility, accommodation, affordability and acceptability (Aday and Andersen, 1974; Penchansky and Thomas, 1981; Joseph and Phillips, 1984). The first two dimensions are spatial in nature and refer to the number of providers and travel impedance (which are not independent of each other and usually need to be combined), while the latter three dimensions are essentially nonspatial and reflect health-care financing arrangements and cultural factors. Availability and accessibility are often fused and referred to as spatial accessibility (Khan, 1992; Guagliardo, 2004). As an effective indicator, potential spatial accessibility (henceforth referred to as spatial accessibility) has been widely used in previous studies to measure access to health care and estimate its equity (Gu et al., 2019; Kaur Khakh et al., 2019; Lu et al., 2019). Identifying areas with limited spatial accessibility could enable planners to understand and optimize the geographical distribution of health-care resources to improve health outcomes (Shah et al., 2016; Smith et al., 2017; Ma et al., 2018; Tao et al., 2018b). Therefore, it is essential to measure spatial accessibility accurately and in detail. 
Spatial accessibility measures are typically classified into four categories: provider-to-population ratios (regional availability); distance to nearest provider; average distance to a set of providers; and gravitation models of provider influence (Joseph and Bantock, 1982; Guagliardo, 2004). In recent years, the two-step floating catchment area (2SFCA) method, one of the gravitation models, has been widely used for measuring spatial accessibility due to ease of understanding and operation (Delamater, 2013; Izumi et al., 2016; Tao et al., 2018a; Chen and Jia, 2019; Delamater et al., 2019). The 2SFCA method computes the provider-population ratio within the catchment area and then sums up these ratios defined by a threshold travel time for each population location, which takes care of the geopolitical border-crossing conundrum (Luo and Wang, 2003). However, an artificial line between accessible and inaccessible areas would be drawn by the original 2SFCA method (Wang, 2012), which means that there is either uniform spatial accessibility within the catchment or no spatial accessibility outside the catchment (Guagliardo, 2004). To address the problem of uniform accessibility within the catchment, an enhanced two-step floating catchment area (E2SFCA) method has been proposed for measuring spatial accessibility by applying weights to different travel time zones to account for distance decay (Luo and Qi, 2009), an approach that has been widely used (Kim et al., 2018; Luo et al., 2018; Zhu et al., 2019). In the E2SFCA method, each distance ring tier within the catchment is assigned a particular tier weight according to the segment values of a continuous distance decay function, such as the Gaussian function, which differentiates distance impedance within the catchment (Luo and Qi, 2009), instead of a dichotomous distance decay function of the traditional 2SFCA method, which assumes that the accessibility of population locations within the catchment is equal (Luo and Wang, 2003).

In order to obtain the values of spatial accessibility, the 2SFCA method family requires three aspects of data: supply, demand and interaction between supply and demand (Langford et al., 2016; Yang and Mao, 2018). In general, supply data include the number of staff, number of beds, locations of institutions, etc., while the demand data are the information of potential patients, often represented by population distribution. Importantly, the interaction between supply and demand is expressed in terms of travel time in this connection (Delamater et al., 2012; Gu et al., 2019). The location of institutions and their number of staff and beds can usually be obtained from the official websites of the institutions or the local health administration departments and the travel time can be calculated based on road networks and/or online maps, whereas high-resolution population distribution data are typically difficult to obtain (Li and Zhou, 2018; Chen et al., 2019; Yang et al., 2019).

The 2SFCA method was first applied in industrialized countries, where the geographic or population-weighted centroid of a census tract could be adopted for defining population locations (Luo and Wang, 2003; Delamater, 2013; Delamater et al., 2019; Kaur Khakh et al., 2019), while the geometry of the county or subdistrict, alternatively a population centroid, was usually used for defining this measure in the developing countries (Tao et al., 2018b; Zhu et al., 2019). Some authors disaggregated the population data at the county or sub-district evenly (Pan et al., 2016; Wang et al., 2018; Ni et al., 2019; Zhang et al., 2019a). However, the population data at these levels are low-resolution, outdated and therefore not possible to compare (Gregory et al., 2010).

In the US, if the population in a census tract is supposed to be concentrated in the population-weighted centroid, the number of people inside the centroid may be overestimated and that outside underestimated. In China, on the other hand, the land areas of counties or sub-districts are usually large, e.g., the average county area is $3376 \mathrm{~km}^{2}$, while the largest cover up to $200,000 \mathrm{~km}^{2}$. County and sub-district population distribution produced by directly averaging of the population in corresponding areas would indicate the assumption that the population within those areas are evenly distributed, which is far from reasonable and lead to biased outcomes of spatial accessibility. To measure population distribution more accurately, a number of research teams have produced simulations of population distribution, such as LandScan (Dobson et al., 2000), WorldPop (Stevens et al., 2015), PoiPop (Ye et al., 2019) and population maps (Bagan and Yamagata, 2015; Li and Zhou, 2018; Chen et al., 2019). These population distribution measures are modelled by professional teams combining a large amount of remotelysensed and geospatial data for all researchers to use. However, they are not very recent and only available for certain years. In addition, as the predicted layers of those are global in scope, the precision may not be high in small-scale areas. Gao et al. (2019) proposed that more accurate night-time light data simulates a more detailed population distribution, and these data are real-time and easier to obtain (Huang et al., 2016; Li and Zhou, 2018; Song et al., 2019). However, the various population distribution measures produce highly variable accuracies depending on which regions they are applied (Li et al., 2018; Luo et al., 2019; Wang et al., 2019). For instance, PoiPop shows a higher accuracy than WorldPop in Beijing, Shanghai, Guangzhou and Chongqing (Ye et al., 2019), while LandScan tends to underestimate people counts in Poland (Calka and Bielecka, 2019), and the LuoJia1-01 simulation data had a higher accuracy than that of Landscan and European Commission's Global Human Settlement population (Gao et al., 2019). The accuracy of the fit was poorer at the community level and at smaller scales (Gao et al., 2019). In health-care studies, especially in those evaluating accessibility of health care, accurate identification of underserved areas is expected, which puts forth a requirement for the accuracy of population distribution (demand data) in specific areas. However, although it is believed that examination of the effect of population distribution measures is imperative, these issues have not been fully explored in health-care studies. The purpose of this manuscript was to compare assessments of spatial accessibility based on several types of common population distribution measures. Specifically, we aimed to answer the following three questions: i) Does spatial accessibility vary in different types of population distribution measures?; ii) Does the identification of underserved areas, which are of great significance for the equity of health-care resources, show different values?; and iii) which is the most appropriate population distribution measure? In order to address these issues, and in consideration of the importance of primary health-care institutions for the establishment of the hierarchical medical system and promotion of human health, a case study was conducted based on primary health-care institutions in Hainan Province, China.

\section{Materials and methods}

\section{Study area and data}

This study focused on the main part of Hainan Province, i.e. Hainan Island, a tropical island in southern China (Figure 1A) covering an area of $33,900 \mathrm{~km}^{2}$ with a total population of $8,409,084$ in 
2018. In 2018, the island included 24 county-level administrative units and 222 township-level administrative units (Figure 1B). The number of primary health-care institutions and their health staff in 2018 can be found in Table 1. The unique physical geography of this place made it ideal for implementing the 2SFCA family for a spatial accessibility study since it would be less affected by healthcare resource allocations in adjacent areas. Primary health-care in China provides basic clinical care and public health services. It includes community health centres/stations in urban areas and township health centres as well as village clinics in rural areas $(\mathrm{Li}$ et al., 2017). These institutions were considered as supply data in this study. Their locations on Hainan Island in 2018 are shown in Figure 1C. On account of the importance of health staff in primary care delivery, the number of health staff was selected as the supply indicator to evaluate the spatial accessibility of primary health-care institutions. Since primary health-care services are provided to all residents, the population distribution of the whole population (see next section) was adopted as the demand data in this study. The road network data was used to measure the travel time between supply and demand. Each road was assigned a speed class value from 1 to 8 (Figure 1D) according to road class, traffic, physical conditions and the highway technical standards of China. Considering that generally neither maximum nor minimum speed are enforced, the median of the speed limit of each class, namely the average of the maximum and minimum values of the limit range, the following speed classes were adopted: $90 \mathrm{~km} / \mathrm{h}, 65$ $\mathrm{km} / \mathrm{h}, 50 \mathrm{~km} / \mathrm{h}, 45 \mathrm{~km} / \mathrm{h}, 35 \mathrm{~km} / \mathrm{h}, 25 \mathrm{~km} / \mathrm{h}, 15 \mathrm{~km} / \mathrm{h}$ and $5 \mathrm{~km} / \mathrm{h}$.

The sources of all data in this study except the demand data are shown in Table 2.

Table 1. Health-care institutions and health professionals on Hainan Island.

\begin{tabular}{lcc} 
Health centre (type) & $\begin{array}{c}\text { Institution } \\
\text { (no.) }\end{array}$ & $\begin{array}{c}\text { Health staff } \\
\text { (no.) }\end{array}$ \\
Community health centres/stations & 177 & 2,861 \\
Township health centre & 299 & 9,109 \\
\hline Village clinic & 2,715 & 4,263 \\
Total & 3,191 & 16,233 \\
\hline
\end{tabular}
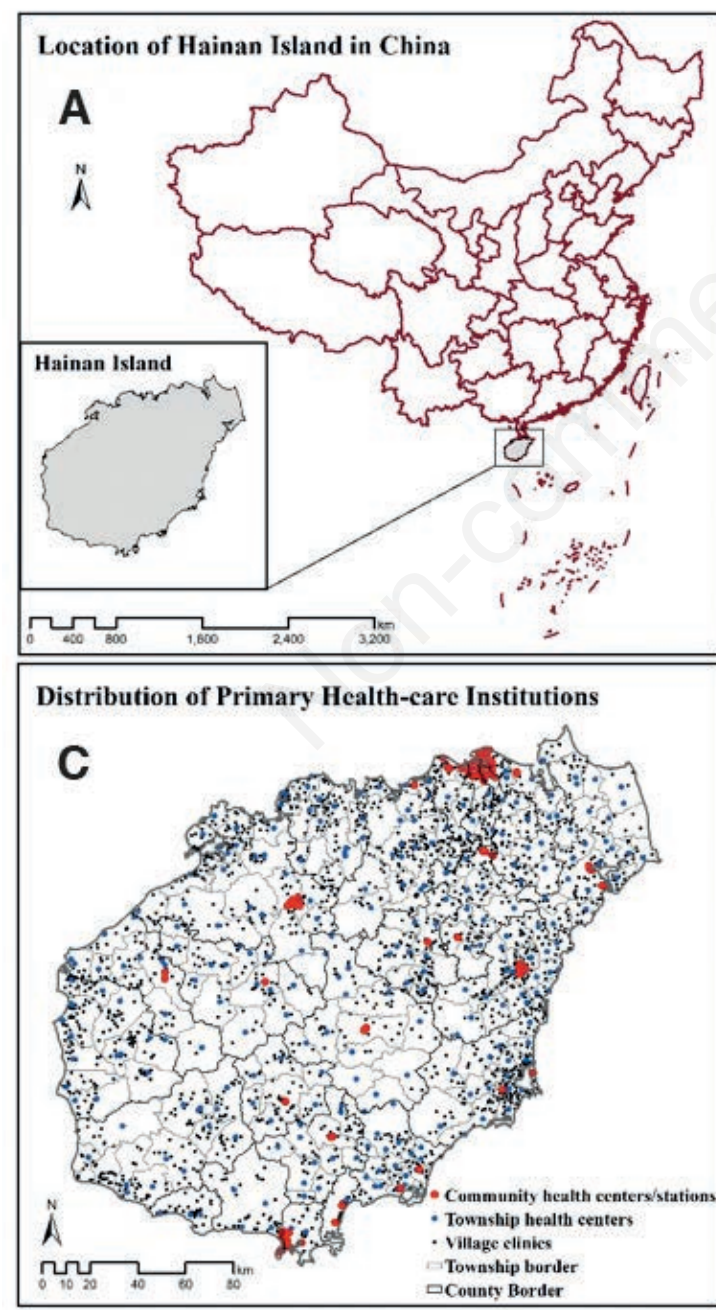
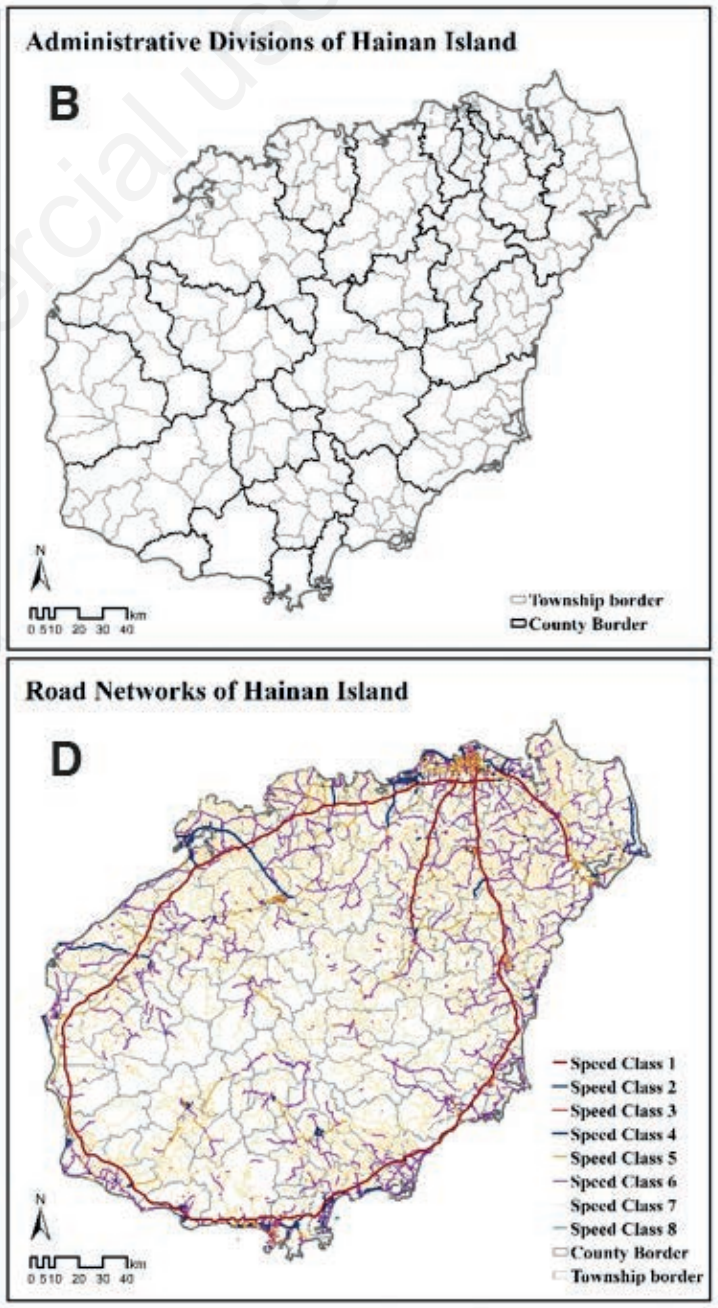

Figure 1. Hainan Island, China: general information. A) The location of Hainan Island in China; B) administrative divisions; C) the distribution of primary health-care institutions; D) road network. 


\section{Population distribution measures}

In China, community health stations in communities and village clinics are the primary level of primary health-care institutions. Communities and villages are administrative divisions below sub-district and township, which are both at the township level. They are usually small in size although some villages are very large, but the population is usually concentrated in a relatively small area around the administrative centre. It was thus assumed that the population at the community/village level on Hainan Island in 2018 would live near the administrative centre of the communities and villages and these centres were regarded to be sub-unit residence locations. This was the first population distribution entity (hereafter referred to as VillagePop) (Figure 2A) in this

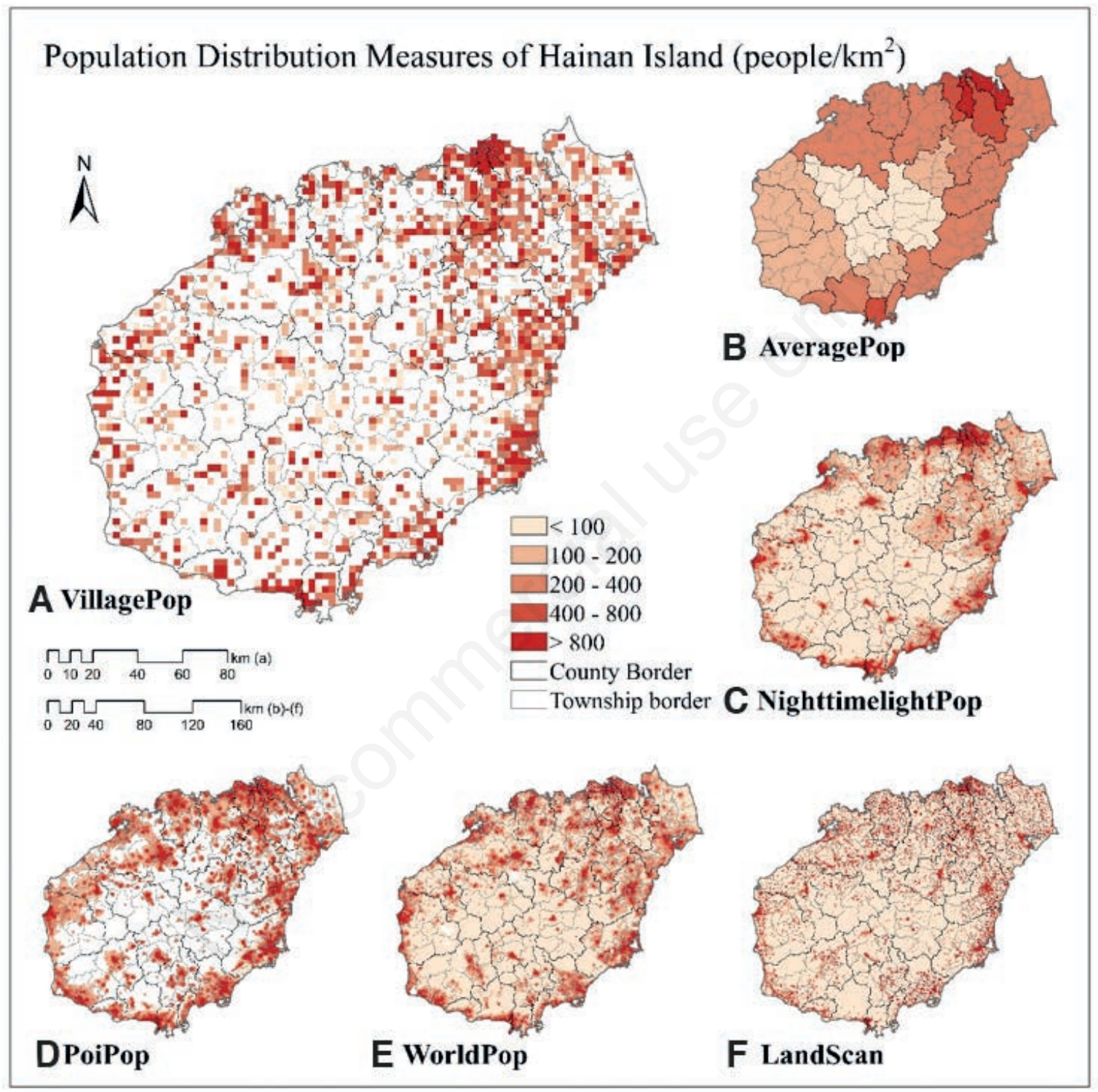

Figure 2. A-F) The six different population distribution measures applied at Hainan Island, China. VillagePop shows the data as point features on a grid system close to $3 \times 3 \mathrm{~km}$ (the total area of Hainan Island $\left(33,900 \mathrm{~km}^{2}\right)$ divided by the number of villages there $(n=3414)$. The others are presented as raster.

Table 2. General data information.

$\begin{array}{lccc}\text { Type of data } & \text { Year } & \text { Specific item } & \text { Data source } \\ \text { Primary health-care institutions } & 2018 & \text { Location and number of health staff } & \text { Hainan Health Commission } \\ \text { Baidu map app }\end{array}$

*Specifically, the National Fundamental Geographic Information System of China, which is the main part of China's NSDI. 
study and it was used as reference for all comparisons (Gaughan et al., 2016; Zhu et al., 2019).

Since population data at the county level are publicly available from Census and Statistic Yearbooks in China, the second population distribution entity was produced by evenly disaggregating the Hainan Island county population data for 2018 into raster made up of a $500 * 500 \mathrm{~m}$ grid (hereafter referred to as AveragePop) (Figure $2 \mathrm{~B})$. The third population data entity was obtained by disaggregating this population data into the $500 * 500 \mathrm{~m}$ raster with the nighttime light intensity as the weight (hereafter referred to as NighttimelightPop) (Figure 2C). This entity simulates the population distribution by recording the light intensity produced by anthropogenic activities (Jiang et al., 2019; Wang et al., 2019). The raster centres were assumed to be the location of an aggregation of residences. The remaining three population distribution data were obtained from LandScan, WorldPop and PoiPop.

LandScan relies on the Global Population Project by Dobson et al. (2000), who produced a worldwide population database at the $30 * 30$ resolution of the coordinate system. In this approach, the best available official census counts are distributed through smart interpolation based on the relative probability coefficients due to road proximity, slope, land cover and night-time lights. The best available official census data are usually collected at the provincial level (one administrative division below the national) and acquired from published sources.

The WorldPop program by Stevens et al. (2015) presents open, contemporary population distribution data with a the $100 * 100 \mathrm{~m}$ resolution using a new semi-automated dasymetric modelling approach that incorporates detailed census and ancillary data in a flexible Random-Forest estimation technique. Country-specific census data are collected from the national institute of statistic of each country, and the ancillary data include a series of widely available, remotely-sensed, geospatial data. The PoiPop (Ye et al., 2019) combines points-of-interest (POIs) with multi-source remotely sensed data in a random-forest model that disaggregates the Chinese 2010 county-level (one administrative division below the province) census population data into $100 * 100 \mathrm{~m}$ raster. The datasets include the radiance-calibrated night-time light product from the defence meteorological satellite program's operational linescan system, the normalized difference vegetation index from the Belgian vlaamse instelling voor technologish onderzoek and POIs retrieved from the Baidu Map, which is the largest desktop and mobile map service provider in China. The raw population data from LandScan, WorldPop and PoiPop are based on census, usually at higher administrative levels. As the estimation layers of those are global in scope, they may be appropriate for researches in large-scale areas, but the precision is generally much lower in small-scale areas. When population data can be obtained from a more recent time period, or at a lower administrative level, the population distribution can be redistributed using the values of the estimation layers as weights. In this study, the estimated values available from the LandScan, WorldPop and PoiPop layers were used as weights to redistribute the county-level population data in Hainan Island for 2018 (Figure 2D-F). The sources of the demand data are shown in Table 3.

\section{The enhanced two-step floating catchment area method}

The E2SFCA method was adopted for preliminary research in this study. In step 1, we calculated a supply-to-demand ratio for each institution based on the total population able accessing it. In step 2, the sum of the ratio scores of institutions within reach of each residence gave us a spatial accessibility score for each residence location, which can be represented mathematically according to Luo and Qi (2009) as:

$$
A_{i}=\sum_{j \in\left\{d_{i j} \in D_{r}\right\}} \frac{S_{j}}{\sum_{k \in\left\{d_{k j} \in D_{r}\right\}} P_{k} W_{r}} W_{r}
$$

where $A_{i}$ is the spatial accessibility of population at residence location $i$ to health staff; $S_{j}$ the number of health staff at institution location ${ }_{j} ; P_{k}$ the population of residence location $k$ falling within the catchment $d_{k j} \in D_{r} ; d_{k j}$ the travel time between $k$ and $j ; d_{i j}$ the travel time between $i$ and ${ }_{j}$, $D_{r}$ the $r^{\text {th }}$ travel time zone $(r \in[1,3])$ within the catchment; and $W_{r}$ the distance weight for $r^{\text {th }}$ travel time zone calculated from the Gaussian function.

Taking into account both the goal of the 15-minute primary health care circle proposed by the Hainan Provincial Government (The People's Government of Hainan Province, 2018) and the literature (Luo and Qi, 2009; Voigtländer and Deiters, 2015; Pan et al., 2016; Wang and Pan, 2016; Wang et al., 2018), three catchment areas of 0-15 min, 15-30 min and 30-60 min were included

Table 3. Demand data.

\begin{tabular}{|c|c|c|c|c|}
\hline $\begin{array}{l}\text { Population distribution } \\
\text { data project }\end{array}$ & Year & Display & Data source & Remark \\
\hline VillagePop & 2018 & Point feature & Hainan Health Commission & Refers to Community/village populations \\
\hline AveragePop & 2018 & $500 \times 500 \mathrm{~m}$ raster & Summed up by VillagePop & Refers to evenly disaggregated county populations \\
\hline NighttimelightPop & 2018 & $500 \times 500 \mathrm{~m}$ raster & $\begin{array}{l}\text { The National Oceanic and Atmospheric } \\
\text { Administration }\end{array}$ & $\begin{array}{l}\text { Used as weight for the disaggregation of the county } \\
\text { population* }\end{array}$ \\
\hline LandScan & 2018 & $1 \times 1 \mathrm{~km}$ raster & The LandScan Project & $\begin{array}{l}\text { Used as weight for the disaggregation of the county } \\
\text { population* }\end{array}$ \\
\hline WorldPop & 2018 & $100 \times 100 \mathrm{~m}$ raster & The WorldPop Program & $\begin{array}{l}\text { Used as weight for the disaggregation of the county } \\
\text { population* }\end{array}$ \\
\hline PoiPop & 2019 & $100 \times 100 \mathrm{~m}$ raster & $\begin{array}{l}\text { Zhejiang University research team } \\
\text { (Ye et al., 2019) }\end{array}$ & $\begin{array}{l}\text { Used as weight for the disaggregation of the county } \\
\text { population* }\end{array}$ \\
\hline
\end{tabular}

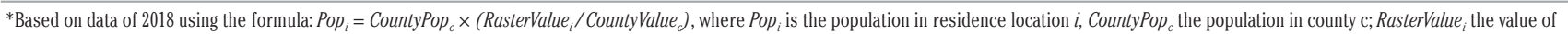
resident location i and CountyValue ${ }_{c}$ the sum of values of residence locations within county c obtained from Night-time light, LandScan, WorldPop and PoiPop, respectively. 
and the weights calculated according to the Gaussian function, which were $0.880,0.316$ and 0.010 respectively. The formula according to Luo and Wang (2003) is the following:

$f(d)=e^{-d^{2} / \beta}$

where $d$ is the threshold travel time; and $\beta$ the distance impedance coefficient, here $=440$ (Wan et al., 2012).

The Service Area function of Network Analyst was implemented in ArcGIS 10.5 (ESRI, Redlands, CA, USA) to calculate the polygons of the three catchment areas for each health-care institutions and residence locations individually in a preliminary exploration.

\section{Statistical approaches}

As the resolutions of the six population distributions are different, the resolutions of the spatial accessibilities calculated by E2SFCA are also different. Since one of the main purposes of research on health-care resources allocation is to provide scientific implications to policy makers and investors, and the lowest level of administrative unit in China is the township level (which is also the lowest level of publicly available administrative boundary data), the spatial accessibility based on six population distribution measures with different resolutions was conveniently aggregated to the township level. The population weighted mean within each town was calculated according to Shen (1998) and Luo and Wang (2003) as follows:

$$
A_{t}^{W}=\frac{\sum_{i=1}^{n} P_{i} A_{i}}{\sum_{i=1}^{n} P_{i}}
$$

where $A_{t}^{w}$ is the weighted spatial accessibility in town $t ; i$ the residence location within town $t ; A_{i}$ the spatial accessibility at the residence location $i$; and $P_{i}$ the population at the residence location $i$.

Differences in spatial accessibility results at the township level derived from each population distribution measure were tested statistically; i) by the two-way analysis of variance (ANOVA) (Mishra et al., 2019) and the Dunnett test (Lee and Lee, 2018) performed to identify the differences using SPSS 20.0 with the traditional $a=0.05$ significance level; and ii) by the root mean square error (RMSE) and the mean absolute error (MAE) to quantify and compare the errors using R 3.6.1 (Dessau and Pipper, 2008). The formulas for calculating RMSE and MAE are the following:

$$
R M S E=\sqrt{\frac{1}{m} \sum_{i=1}^{m}\left(A_{t}^{\text {Wlog }}-A_{t_{0}}^{\text {Wog }}\right)^{2}}
$$

$$
M A E=\frac{1}{m} \sum_{i=1}^{m}\left|A_{t}^{\text {Wlog }}-A_{t_{0}}^{\text {Wlog }}\right|
$$

where $A_{t}^{\text {Wlog }}$ is the $\log _{e}$ scale of the weighted accessibility of each of the last five population distribution measures; and $A_{t 0}{ }^{\text {Wlog }}$ the $\log _{e}$ scale of the weighted accessibility of VillagePop.

\section{Results}

\section{Spatial accessibility}

Maps of the E2SFCA results based on the six population distribution measures are depicted in Figure 3 (the residence level) and Figure 4 (the township level). The spatial accessibility was found to be highly uneven throughout the island, with higher levels in coastal areas and areas along roads. In addition, the spatial accessibility based on the six population distribution measures appeared to be different at these two levels.

Summary statistics for the six results are found in Table 4 . Since the accessibility had a skewed distribution, the median was calculated to describe the degree of concentration and the minimum, maximum and inter-quartile range to describe the degree of dispersion. At the residence level, the median spatial accessibility based on VillagePop was higher than other population distribution measures, but the maximum and inter-quartile ranges were the lowest. While at the township level the median spatial accessibility based on VillagePop was near those of the other population distribution measures, the inter-quartile range was lower than those of the other population measures, except WorldPop whose difference was nearly 0.01 . At both the residence and the township level, the maximum values of spatial accessibility based on LandScan,

\begin{tabular}{|c|c|c|c|c|c|c|c|c|}
\hline \multirow[t]{2}{*}{$\begin{array}{l}\text { Population distribution } \\
\text { data project }\end{array}$} & \multicolumn{4}{|c|}{$\begin{array}{l}\text { Spatial accessibility at the residence } \\
\text { location level }\end{array}$} & \multicolumn{4}{|c|}{$\begin{array}{l}\text { Spatial accessibility } \\
\text { at the township level }\end{array}$} \\
\hline & Median & Min. & Max. & IQR & Median & Min. & Max. & IQR \\
\hline VillagePop & 1.73 & 0.00 & 29.70 & 1.44 & 1.88 & 0.45 & 7.16 & 1.00 \\
\hline AveragePop & 1.00 & 0.00 & 38.03 & 2.48 & 1.97 & 0.36 & 8.57 & 2.00 \\
\hline NighttimelightPop & 1.06 & 0.00 & 66.07 & 2.16 & 2.07 & 0.48 & 7.00 & 1.18 \\
\hline LandScan & 0.95 & 0.00 & 1668.44 & 1.90 & 1.99 & 0.35 & 24.19 & 1.32 \\
\hline WorldPop & 0.95 & 0.00 & 515.17 & 1.81 & 1.97 & 0.56 & 6.34 & 1.01 \\
\hline PoiPop & 1.30 & 0.00 & 2483.78 & 1.53 & 2.08 & 0.00 & 2483.78 & 1.35 \\
\hline
\end{tabular}
WorldPop and PoiPop were found to be much higher than that of

Table 4. Summary statistics of spatial accessibility to primary health care.

$\mathrm{IQR}$, inter-quartile range. 
VillagePop, except for the maximum value of WorldPop at the township level, which was lower than that of VillagePop.

\section{Descriptive comparison of differences: scatter plots and maps}

Figures 5 and 6 illustrate the relationships of spatial accessibility at the township level of the six population distribution measures. As can be seen in Figure 5, all the population distribution measures investigated compared with VillagePop both over- and under-estimate spatial accessibility as points fall both above and below the 1:1 dashed line.

The differences among the spatial accessibility of health staff per 1000 people based on the five population distribution measures subtracted from the VillagePop is shown in Figure 6. The variation within 0.5 health staff per 1000 people in relation to VillagePop was relatively small. Compared with the spatial accessibility revealed by VillagePop in Figures 4 and 5, the other population distribution measures demonstrated both over-estimated and under-estimated values. Specifically, more areas with under-estimated spatial accessibility were presented by AveragePop (Figure $6 \mathrm{~A}$ ) and more areas with over-estimated spatial accessibility found by PoiPop (Figure 6C).

\section{Identification of the differences}

In order to identify whether the differences were statistically significant by performing the two-way ANOVA and the Dunnett

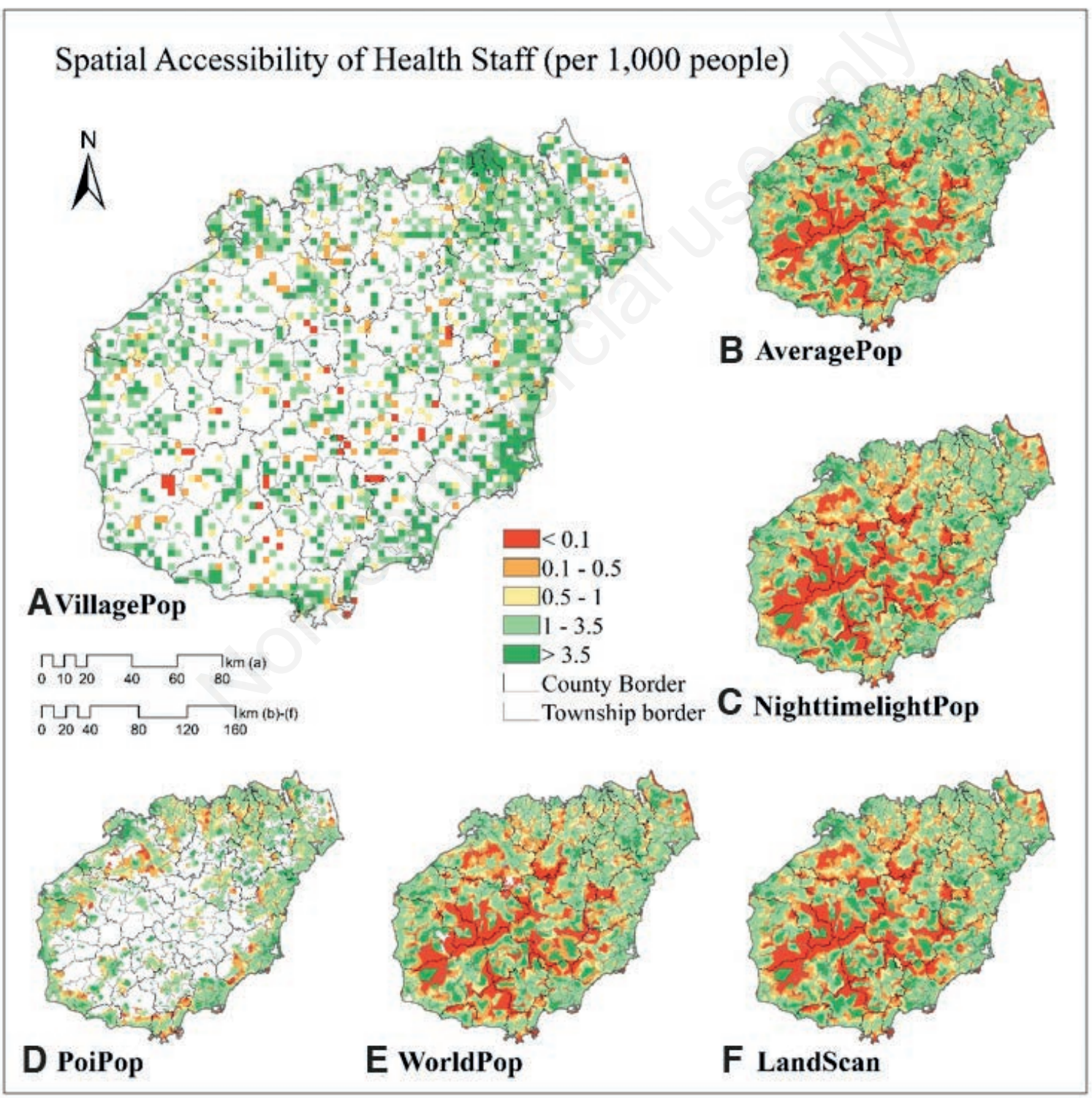

Figure 3. A-F) Spatial accessibility to primary health care at the residence level on Hainan Island, China. VillagePop shows the data as point features on a grid system close to $3 \times 3 \mathrm{~km}$ (the total area of Hainan Island $\left(33,900 \mathrm{~km}^{2}\right)$ divided by the number of villages there $(n=3414)$. The others are presented as raster. 
test, the weighted accessibility of various population distribution measures at the township level were converted to the $\log _{e}$ scales to satisfy the normality:

$A_{t}^{W \log }=\log _{e}\left(A_{t}^{W}+1\right)$

where $A_{t}^{\text {Wlog }}$ is the $\log _{e}$ scale and $A_{t}^{W}$ is calculated by Eqs. 1 and 3.

Tables 5 and 6 report the results of the two-way ANOVA and the Dunnett test at the $\log _{e}$ scale with the traditional $a=0.05$ significance level. The results of the two-way ANOVA $(\mathrm{P}<0.001)$ test revealed that the spatial accessibilities given by the six population distribution measures were not all the same. Further pair-wise comparisons by the Dunnett test (using VillagePop as the control) demonstrated that the mean values were significantly different for AveragePop $(\mathrm{P}<0.01)$ and PoiPop $(\mathrm{P}<0.001)$, while they were not for the other measures suggesting that the spatial accessibility values revealed by AveragePop and PoiPop were considerably higher than that of VillagePop.

\section{Quantification of the differences}

RMSE and the MAE were calculated to quantify the various values of the spatial accessibility given by the population distribution measures. As can be seen in Table 7, the PoiPop showed the largest difference (166.23 and 12.27, respectively) followed by AveragePop (2.17 and 1.46, respectively), which corroborates the results in Figure 6.

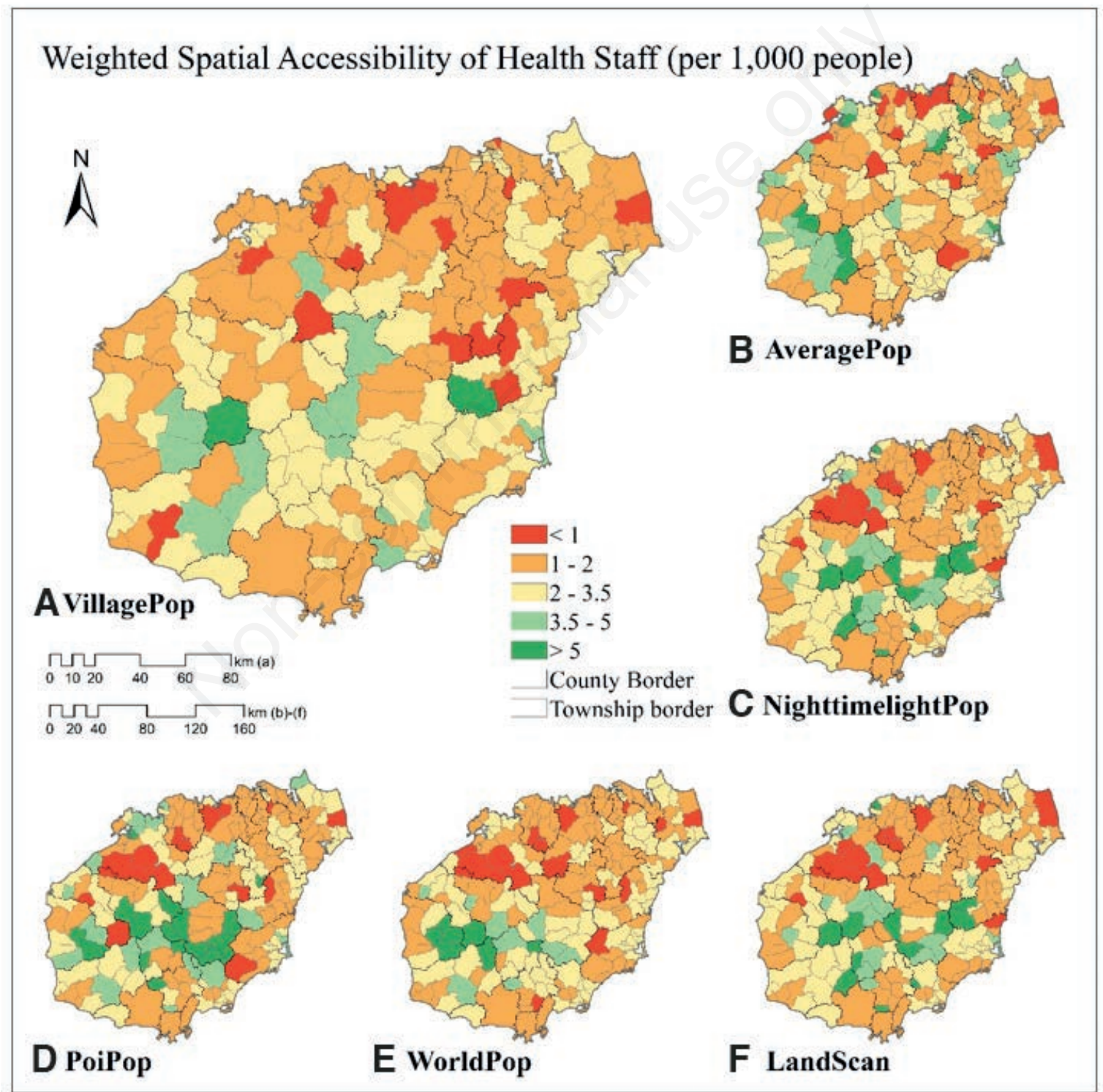

Figure 4. A-F) Weighted spatial accessibility to primary health care at the township level on Hainan Island, China. VillagePop shows the data as point features on a grid system close to $3 \times 3 \mathrm{~km}$ (the total area of Hainan Island $\left(33,900 \mathrm{~km}^{2}\right)$ divided by the number of villages there $(n=3414)$. The others are presented as raster. 
Table 5. Spatial accessibility of population distribution measures: differences according to the two-way ANOVA test.

\begin{tabular}{lcccc} 
& df & Mean square & P & \\
Population distribution & 5.000 & 0.563 & 4.844 & $<0.001$ \\
Town & 221.000 & 0.454 & 3.908 & $<0.001$ \\
\hline
\end{tabular}

df, degree of freedom; F, test statistic.

Table 6. Spatial accessibility of population distribution measures: differences according to the Dunnett test.

\begin{tabular}{|c|c|c|c|c|c|}
\hline \multirow{2}{*}{$\begin{array}{l}\text { Population distribution } \\
\text { data project }\end{array}$} & \multirow{2}{*}{ Mean difference } & \multirow{2}{*}{ Standard error } & \multirow[t]{2}{*}{ P-value } & \multicolumn{2}{|c|}{$95 \%$ confidence interval } \\
\hline & & & & Lower bound & Upper bound \\
\hline VillagePop* & - & - & - & - & - \\
\hline AveragePop & 0.1010 & 0.3235 & 0.008 & 0.0197 & 0.1824 \\
\hline NighttimelightPop & 0.0719 & 0.3235 & 0.104 & -0.0095 & 0.1532 \\
\hline LandScan & 0.0729 & 0.3235 & 0.096 & -0.0085 & 0.1543 \\
\hline WorldPop & 0.0281 & 0.3235 & 0.855 & -0.0533 & 0.1095 \\
\hline PoiPop & 0.1411 & 0.3235 & $<0.001$ & 0.0597 & 0.2224 \\
\hline
\end{tabular}

*Set as control when running the operation.

A

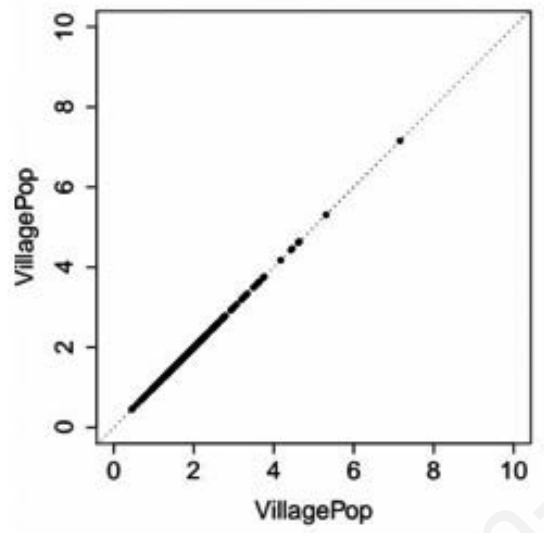

D

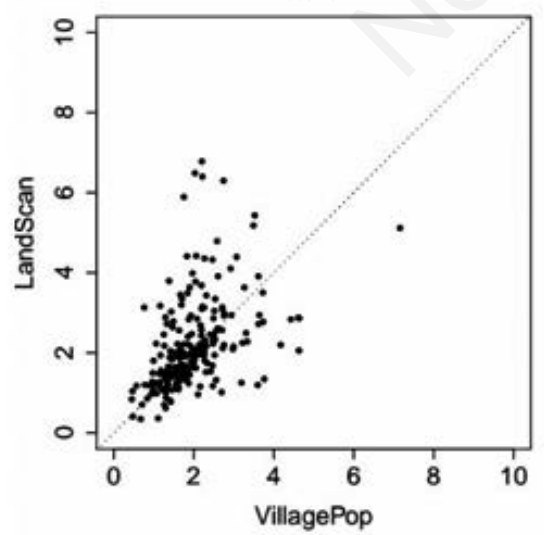

B

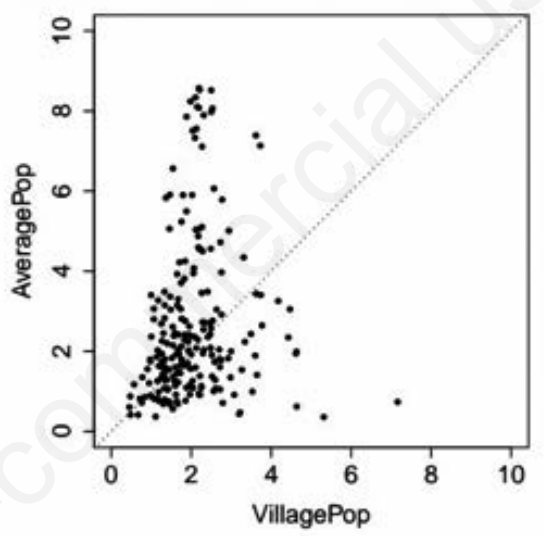

E

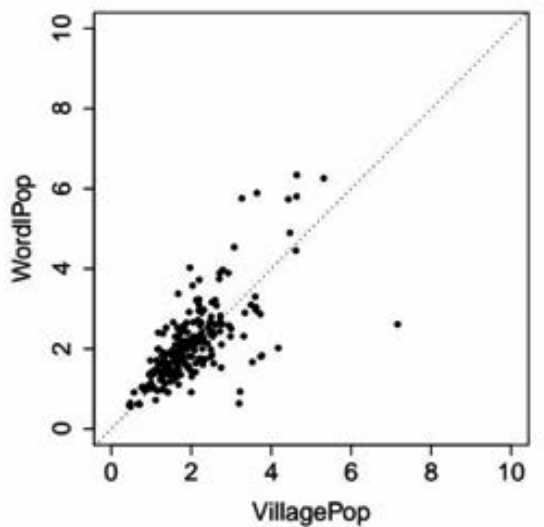

C

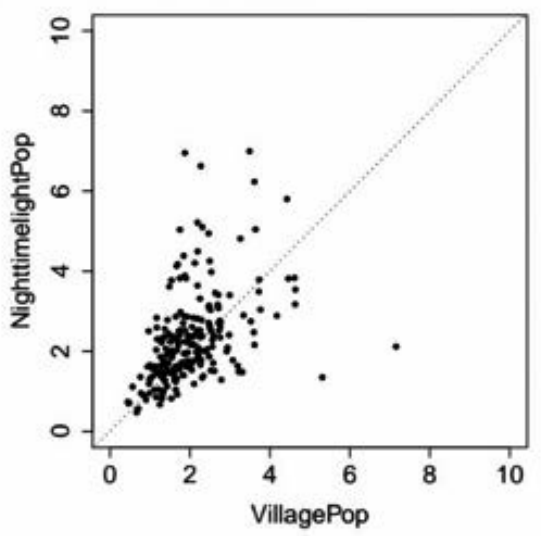

F

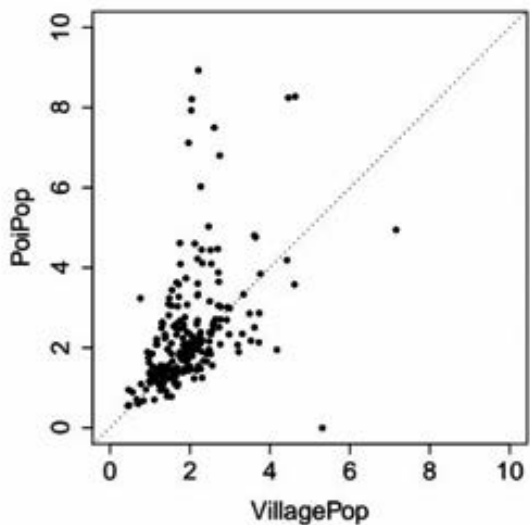

Figure 5. A-F) Scatterplots of the spatial accessibility to primary health care based on the six population distribution measures at the township level. Each data point corresponds to a township with the spatial accessibility of VillagePop (the horizontal axis) compared to the six population distribution measures (the vertical axis). For each type of population distribution measure, the situation of points below the dashed line indicates a lower spatial accessibility compared to VillagePop, while situations above the line means the opposite, i.e. a higher spatial accessibility compared to VillagePop. 


\section{Identification of underserved areas}

With reference to the outline of the Chinese National Health Service System Plan 2015-2020 (General Office of the State Council, PRC, 2015), areas where the spatial accessibility to primary health-care is less than 3.5 health staff per 1000 people should be considered under-served (UAs). The UAs at the township level are shown in Figure 4 and Table 8. In Figure 4, the red, orange and yellow polygons indicate the towns where the weighted spatial accessibility was lower than 3.5 health staff per 1000 people, while the light green and dark green polygons show those where it was higher. It can be seen that the spatial accessibility of most areas of Hainan Island is lower than 3.5 health staff per 1000 people.

Table 8 expresses the differences in the identification of the UAs of the various population distribution measures. Likewise, most areas were identified as UAs in each population distribution measure, especially in VillagePop and WorldPop, where up to $93 \%$ of the towns were identified as UAs. The population of identified UAs based on the six population distribution measures were all more than $90 \%$ of the total population and the highest was $98 \%$ of the values given by WorldPop. Taking VillagePop as the reference, the unrecognized UAs (unUAs) were defined as areas accounted as UAs in the reference but not recognized as such in relation to the other five population distribution measures. Likewise, misidenti-

Table 7. Comparison and quantification of errors related to differences in the spatial accessibility results at the township level.

\begin{tabular}{lcc}
$\begin{array}{l}\text { Population distribution } \\
\text { data project }\end{array}$ & RMSE & MAE \\
VillagePop* & - & - \\
AveragePop & 2.17 & 1.46 \\
\hline NighttimelightPop & 1.13 & 0.74 \\
LandScan & 2.12 & 0.92 \\
\hline WorldPop & 0.75 & 0.50 \\
PoiPop & 166.53 & 12.27 \\
\hline
\end{tabular}

*Set as control when running the operation. RMSE, root mean square error; MAE, mean absolute error.

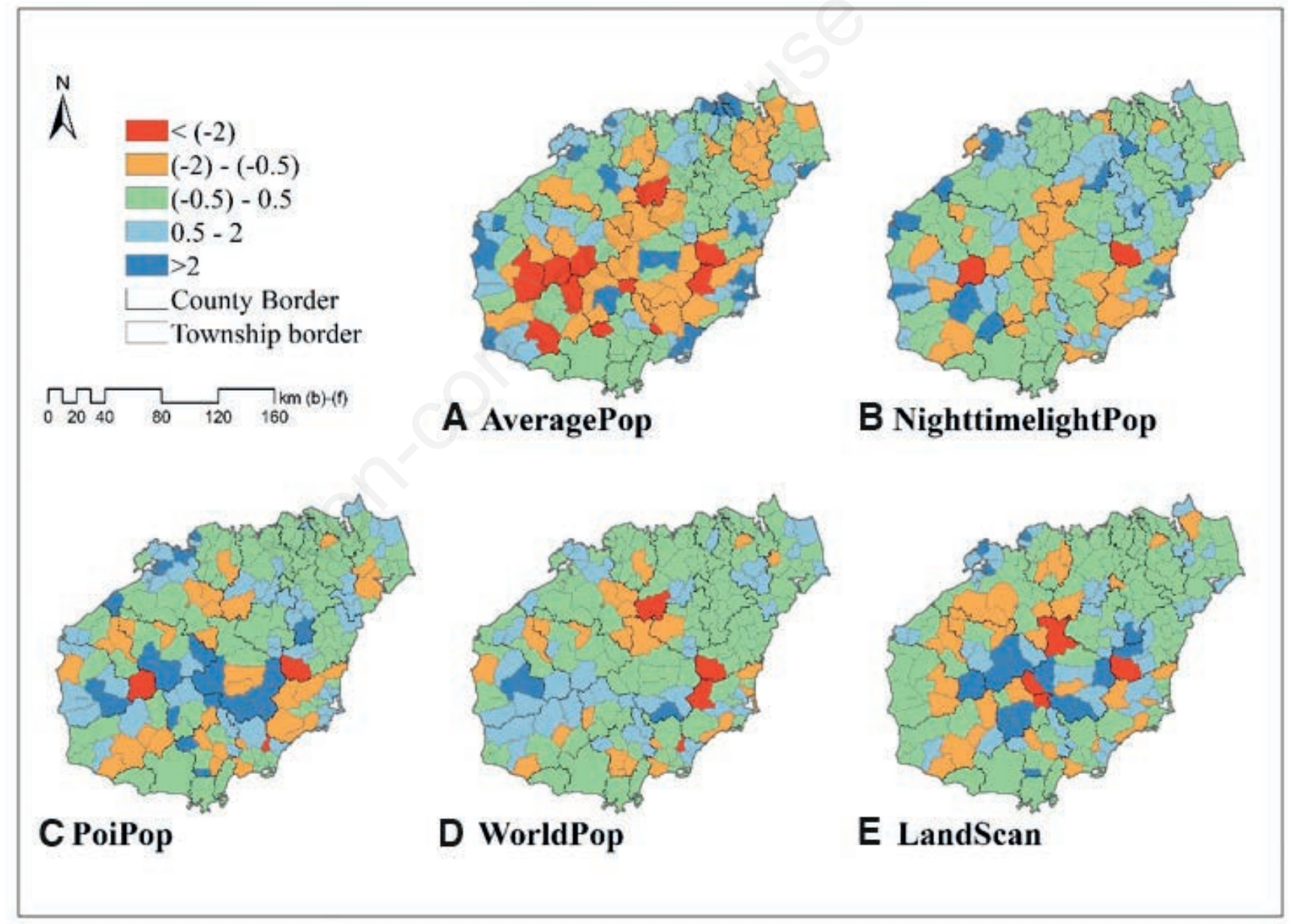

Figure 6. A-E) Variations in spatial accessibility to primary healthcare data produced by five population distribution measures compared with that produced by VillagePop. The figure presents the variation of spatial accessibility data compared with VillagePop, with the data from this measure subtracted to show the difference. The light blue and dark blue denote areas where the number of health staff accessible per 1000 people is at least 0.5 higher than that of VillagePop; the orange and red denote areas where the number of health staff accessible per 1000 people is at least 0.5 lower than that of VillagePop; while green denote areas where the variation of accessibility to health staff per 1000 people compared with VillagePop is within plus or minus 0.5. 
fied UAs (misUAs) were areas not accounted as UAs in the reference but identified as such in relation to the other five population distribution measures. The findings indicate that AveragePop and PoiPop produced more unUAs (up to $20 \%$ and $12 \%$ of towns, respectively), while AveragePop produced more misUAs, up to $6 \%$ of the towns.

\section{Discussion}

In this study, the spatial accessibility to health care has been evaluated based on different population distribution measures. Compared with VillagePop, the difference and the error rates for identification of UAs of AveragePop and PoiPop were larger, while those of NighttimelightPop, LandScan and WorldPop were smaller.

\section{Overall trends}

Although the spatial accessibility based on the six population distribution measures varied widely, the overall trend was consistent. In agreement with a previous study which employed an optimized 2SCFA for measuring spatial accessibility of medical clinics in Montreal Island, Canada, which found medical clinics to be clustered in the central part of the island, where most people also live (Ngui and Apparicio, 2011), we also found an imbalanced distribution of health care facilities. The distribution of health-care institutions in Hainan Island was found to be highly uneven with higher spatial accessibility scores along the coast and road networks, which is consistent with the trend of population distribution. Indeed, the overall spatial accessibility in most areas of Hainan Island is lower than 3.5 health staff per 1000 people, which does not yet meet the national planning requirements. Thus, the vast majority of people there lack sufficient accessibility of primary health-care resources.

The medians of spatial accessibility of primary health staff per 1000 people based on VillagePop was found to be 1.73 at the residence location level and 1.88 at the township level, which meant that the spatial accessibility of half areas were less than 1.73 at the residence location level or 1.88 at the township level. Furthermore, $93 \%$ of the townships were UAs, together covering up to $95 \%$ of the total population. A sampling investigation about the economic burden of diabetes mellitus in Hainan reported that the economic burden posed by medical costs required for the treatment of diabetes in Hainan is massive, with the total medical expenses for this treatment in 2016 amounting to 242.17 billion CNY (about 36.8 billion USD). Only $14.7 \%$ was spent in primary health-care insti- tutions indicating that primary health-care institutions failed to fully achieve their role as gatekeepers (Dong et al., 2019). This might have been induced by the lack of adequate primary healthcare resources in most areas of Hainan. In other areas of China, such as Donghai County in Jiangsu Province, most villages were also situated in UAs (Hu et al., 2013). However, in Shenzhen, one of the fastest growing cities in China, $99.9 \%$ of the residents are able to access the nearest general hospital within 30 minutes ( $\mathrm{Zhu}$ et al., 2019) and in Sichuan, the 5th largest province with a middle level of economic development, $92.3 \%$ of the population are able to access the closest township or community health centres (Wang et al., 2018).

\section{Results diversity}

The previously stated results validate that the estimates of spatial accessibility varied with different population distribution measures, among which those of AveragePop and PoiPop were statistically significant $(\mathrm{P}<0.01$ and $\mathrm{P}<0.001$, respectively. In the quantitative comparison of differences between VillagePop and the other five population distribution measures and were distinct. However, among these, AveragePop and PoiPop displayed larger errors. In addition, there were also disparities in the identification of UAs. Similarly, the error rates of AveragePop and PoiPop were higher and identified more unrecognized UAs. Indeed, the correct identification of UAs is the fundamental purpose of spatial accessibility research (Nakamura et al., 2017), which means that AveragePop and PoiPop would be inappropriate for spatial accessibility research. Comparatively, NighttimelightPop, LandScan and WorldPop, each of which were shown to have lower error rates, were more suitable.

The reasons for the differences between AveragePop and PoiPop are based on the assumptions made about the population distributions. If the population of a county were evenly distributed within the county (as assumed by AveragePop), the population of the sparsely populated area there would be overestimated and those of the densely populated area underestimated, thus showing the inaccurate assessment of spatial accessibility. In PoiPop, there were two townships with abnormal population estimates, one with 0 and one with 4.88 , which led to two outliers of spatial accessibility, one with an abnormally low value $(0)$ and one with an abnormally high value (2483.78). These results could be due to the typically low accuracy at small scales of open databases, and they contributed negatively to the difference outcomes of the test as well as to the difference comparison. NighttimelightPop, LandScan and WorldPop combined a variety of remotely sensed

Table 8. Comparison of underserved areas at the township level.

\begin{tabular}{|c|c|c|c|c|c|c|c|c|}
\hline $\begin{array}{l}\text { Population distribution } \\
\text { data project }\end{array}$ & $\begin{array}{l}\text { UA } \\
\text { (no.) }\end{array}$ & $\begin{array}{l}\text { Proportion } \\
\text { of all towns } \\
\qquad(\%)\end{array}$ & $\begin{array}{l}\text { Population } \\
\text { of all UAs } \\
\text { (no.) }\end{array}$ & $\begin{array}{c}\text { Proportion of } \\
\text { all population } \\
\qquad \%)\end{array}$ & $\begin{array}{l}\text { UnUA } \\
\text { (no.) }\end{array}$ & $\begin{array}{c}\text { Proportion } \\
\text { of all towns } \\
\qquad \%)\end{array}$ & $\begin{array}{l}\text { MisUA } \\
\text { (no.) }\end{array}$ & $\begin{array}{c}\text { Proportion of } \\
\text { all towns } \\
(\%)\end{array}$ \\
\hline VillagePop* & 206 & 93 & 7949067 & 95 & - & - & - & - \\
\hline AveragePop & 175 & 79 & 7587296 & 90 & 45 & 20 & 14 & 6 \\
\hline NighttimelightPop & 194 & 87 & 7935946 & 94 & 21 & 9 & 9 & 4 \\
\hline LandScan & 194 & 87 & 7983316 & 95 & 22 & 10 & 10 & 5 \\
\hline WorldPop & 206 & 93 & 8271502 & 98 & 9 & 4 & 9 & 4 \\
\hline PoiPop & 185 & 83 & 7974659 & 95 & 27 & 12 & 6 & 3 \\
\hline
\end{tabular}

*Set as control when running the operation. UA, underserved areas; UnUA, unrecognized UA; MisUA, misidentified UA. The total number of towns was 222; and the total population was 8,409,084. 
geospatial data showing relatively small differences and they were ultimately closer to the real situation.

The population distribution measures are important factors for assessing spatial accessibility, but they generate differences in spatial accessibility since they are generated by different models based on various data sources. As can be seen from the tests results of this study, these differences were all statistically significant $(\mathrm{P}<0.001$ in two-way ANOVA, while they only reached $\mathrm{P}<0.01$ for AveragePop and $\mathrm{P}<0.001$ for PoiPop in the Dunnett test) and also were different with respect to RMSE, which varied from 0.75 to 166.53 , and MAE, which varied from 0.50 to 12.27 . The spatialization of the permanent resident population based on the township population data and night-time light images showed the distribution and aggregation of the population within administrative areas, while the population density distribution based on the census statistics could only show the population distribution among the administrative areas (Zhang et al., 2019b). Due to the heterogeneity of the population distribution and various dynamic spatial behavioural patterns among the residents, errors for the estimation of spatial accessibility would inevitably appear (Mao and Nekorchuk, 2013).

\section{Recommendation of population distribution measures}

High-precision population data for all types of spatial accessibility are mostly not available (Mao and Nekorchuk, 2013), so researchers must often estimate such data by simulations based on public population databases. NighttimelightPop is clearly better than AveragePop, both theoretically and statistically, as echoed by many researches (Huang et al., 2016; Gao et al., 2019; Kong et al., 2019). Additionally, night-time light data are real-time and relatively easy to obtain. This means that in the absence of real highresolution population distribution data, the weighted average based on night-time light intensity is more appropriate than simply averaging. However, the accuracy of the data available vary greatly as they generate diverse accuracy levels of spatial accessibility in different regions. In this study, LandScan and WorldPop were more precise than PoiPop on Hainan Island, while in the study by Ye et al. (2019), PoiPop showed higher accuracy than WorldPop in Beijing, Shanghai, Guangzhou and Chongqing. LandScan tends to underestimate people counts in Poland (Calka and Bielecka, 2019), which suggests that open data may not be reliable, even in the developed world, except within specific areas. Additionally, public population databases such as LandScan and WorldPop have been available for many years and have usually become outdated and do not therefore always be suitable for the studies using them. Consequently, these databases may not be appropriate for specific spatial accessibility studies, while weighted average population distribution with night-time light intensity as the weight value would be the most appropriate though studies may be limited to specific years and regions.

\section{Limitations and prospects}

There are several limitations of this study which are expected to be addressed in future work. Firstly, for population distribution measures, only community/village population data were taken as the referenced population distribution. Due to the absence of data from the administrative boundary of the community/village, the measure could only assume the administrative centre of the community/village as the residence location. This could be resolved with availability of higher resolution data. Besides, only five population distribution measures were considered to reflect the diver- sity of spatial accessibility and more precise population distribution measures were not included. Secondly, for the calculation of spatial accessibility, only one common measure and the Service Area function of Network Analyst in ArcGIS were used to make a preliminary exploration. Optimized measures and computation approaches might be adopted in future studies in order to enhance the accuracy of the findings. In addition, since the resolution of various population distribution measures were different (done to facilitate the statistical comparison), the spatial accessibility based on different population distribution measures was aggregated to the township level with lower resolutions, which induced loss of data information. Thirdly, the particular location of the study area may need further consideration for the extrapolation of data.

Exploration of the effects of population distribution in other regions or more simple and effective population distribution measures is warranted in future research. More measures and operationalization of spatial accessibility should be considered and simulated data could be used to assess which population distribution measures would be appropriate for specific types of areas.

\section{Conclusions}

Considering the unreliability and limits of available public population databases and the availability and real-time night-time light data, it is recommended to use the weighted average population distribution with night-time light intensity in the future. As the spatial accessibility of primary health-care institutions in Hainan Island was measured, the results of this study could provide useful guidance for policy making in Hainan leading to optimization of the allocation of primary health-care resources.

\section{References}

Aday LA, Andersen R, 1974. A framework for the study of access to medical care. Health Serv Res 9:280-20.

Bagan H, Yamagata Y, 2015. Analysis of urban growth and estimating population density using satellite images of nighttime lights and land-use and population data. GISci Remote Sens 52:765-80.

Calka B, Bielecka E, 2019. Reliability analysis of landscan gridded population data. The Case Study of Poland. IJGI 8:222.

Chen J, Fan W, Li K, Liu X, Song M, 2019. Fitting Chinese cities' population distributions using remote sensing satellite data. Ecol Indic 98:327-33.

Chen X, Jia P, 2019. A comparative analysis of accessibility measures by the two-step floating catchment area (2SFCA) method. Int J Geogr Inf Sci 33:1739-58.

Delamater PL, 2013. Spatial accessibility in suboptimally configured health care systems: a modified two-step floating catchment area (M2SFCA) metric. Health Place 24:30-43.

Delamater PL, Messina JP, Shortridge AM, Grady SC, 2012. Measuring geographic access to health care: raster and network-based methods. Int J Health Geogr 11:15.

Delamater PL, Shortridge AM, Kilcoyne RC, 2019. Using floating catchment area (FCA) metrics to predict health care utilization patterns. BMC Health Serv Res 19:144.

Dessau RB, Pipper CB, 2008. ["R"-project for statistical computing]. Ugeskr Laeger 170:328-30. 
Dobson JE, Bright EA, Coleman PR, Durfee RC, Worley BA, 2000. LandScan: a global population database for estimating populations at risk. Photogramm Eng Remote Sens 66:849-57.

Dong Y, Liu C, Zhou P, Zhu Y, Tang Q, Wang S, Wang X, 2019. How serious is the economic burden of diabetes mellitus in Hainan province? a study based on "System of Health Account 2011". Diabetes Ther 10:2251-63.

Gao N, Li F, Zeng H, Bilsen D van, De Jong M, 2019. Can more accurate night-time remote sensing data simulate a more detailed population distribution? Sustainability 11:4488.

Gaughan AE, Stevens FR, Huang Z, Nieves JJ, Sorichetta A, Lai S, Ye X, Linard C, Hornby GM, Hay SI, Yu H, Tatem AJ, 2016. Spatiotemporal patterns of population in mainland China, 1990 to 2010. Sci Data 3:160005.

General Office of the State Council, PRC, 2015. The Outline of the National Health Service System Plan (2015-2020). Available from: http://www.gov.cn/zhengce/content/2015-03/30/content 9560.htm Accessed: 22 November 2019.

Gregory IN, Marti-Henneberg J, Tapiador FJ, 2010. Modelling long-term pan-European population change from 1870 to 2000 by using geographical information systems. J R Stat Soc Ser A 173:31-50.

Gu X, Zhang L, Tao S, Xie B, 2019. Spatial Accessibility to healthcare services in metropolitan suburbs: the case of Qingpu, Shanghai. Int J Environ Res Public Health 16:225.

Guagliardo MF, 2004. Spatial accessibility of primary care: concepts, methods and challenges. Int J Health Geogr 3:3.

Hu R, Dong S, Zhao Y, Hu H, Li Z, 2013. Assessing potential spatial accessibility of health services in rural China: a case study of Donghai County. Int J Equity Health 12:35.

Huang Q, Yang Y, Li Y, Gao B, 2016. A simulation study on the urban population of China based on nighttime light data acquired from DMSP/OLS. Sustainability 8:521.

Izumi K, Kawatsu L, Ohkado A, Uchimura K, Kato S, 2016. Evaluating the impact of health resource reconstruction on improving spatial accessibility of tuberculosis care. Int $\mathrm{J}$ Tuberc Lung Dis 20:1501-8.

Jiang S, Li J, Duan P, Wei Y, 2019. An image layer difference index method to extract light area from NPP/VIIRS nighttime light monthly data. Int J Remote Sens 40:4839-55.

Joseph AE, Bantock PR, 1982. Measuring potential physical accessibility to general practitioners in rural areas: A method and case study. Social Sci Med 16:85-90.

Joseph AE, Phillips DR, 1984. Accessibility and utilization: geographical perspectives on health care delivery. Harper \& Row, New York, NY, USA.

Kaur Khakh AK, Fast V, Shahid R, 2019. Spatial accessibility to primary healthcare services by multimodal means of travel: synthesis and case study in the City of Calgary. IJERPH 16:170.

Khan AA, 1992. An integrated approach to measuring potential spatial access to health care services. Socioecon Plann Sci 26:275-87.

Kim Y, Byon Y-J, Yeo H, 2018. Enhancing healthcare accessibility measurements using GIS: A case study in Seoul, Korea. PLoS One 13:e0193013.

Kong W, Cheng J, Liu X, Zhang F, Fei T, 2019. Incorporating nocturnal UAV side-view images with VIIRS data for accurate population estimation: a test at the urban administrative district scale. Int J Remote Sens 40:8528-46.

Langford M, Higgs G, Fry R, 2016. Multi-modal two-step floating catchment area analysis of primary health care accessibility. Health Place 38:70-81.

Lee $\mathrm{S}$, Lee DK, 2018. What is the proper way to apply the multiple comparison test? Korean J Anesthesiol 71:353-60.

Li K, Chen Y, Li Y, 2018. The random forest-based method of fineresolution population spatialization by using the international space station nighttime photography and social sensing data. Remote Sens 10:1650.

Li X, Lu J, Hu S, Cheng K, De Maeseneer J, Meng Q, Mossialos E, Xu DR, Yip W, Zhang H, Krumholz HM, Jiang L, Hu S, 2017. The primary health-care system in China. Lancet 390:2584-94

Li X, Zhou W, 2018. Dasymetric mapping of urban population in China based on radiance corrected DMSP-OLS nighttime light and land cover data. Sci Total Environ 643:1248-56.

Lu C, Zhang Z, Lan X, 2019. Impact of China's referral reform on the equity and spatial accessibility of healthcare resources: a case study of Beijing. Soc Sci Med 235:112386.

Luo J, Chen G, Li C, Xia B, Sun X, Chen S, 2018. Use of an E2SFCA method to measure and analyse spatial accessibility to medical services for elderly people in Wuhan, China. IJERPH 15:1503.

Luo W, Qi Y, 2009. An enhanced two-step floating catchment area (E2SFCA) method for measuring spatial accessibility to primary care physicians. Health Place 15:1100-7.

Luo W, Wang FH, 2003. Measures of spatial accessibility to health care in a GIS environment: synthesis and a case study in the Chicago region. Environ Plan B-Plan Des 30:865-84.

Luo P, Zhang X, Cheng J, Sun Q, 2019. Modeling population density using a new index derived from multi-sensor image data. Remote Sens 11:2620.

Ma L, Luo N, Wan T, Hu C, Peng M, 2018. An improved healthcare accessibility measure considering the temporal dimension and population demand of different ages. IJERPH 15:2421.

Mao L, Nekorchuk D, 2013. Measuring spatial accessibility to healthcare for populations with multiple transportation modes. Health Place 24:115-22.

Mishra P, Singh U, Pandey CM, Mishra P, Pandey G, 2019. Application of student's t-test, analysis of variance, and covariance. Ann Card Anaesth 22:407-11.

Nakamura T, Nakamura A, Mukuda K, Harada M, Kotani K, 2017. Potential accessibility scores for hospital care in a province of Japan: GIS-based ecological study of the two-step floating catchment area method and the number of neighborhood hospitals. BMC Health Serv Res 17:438.

Ngui AN, Apparicio P, 2011. Optimizing the two-step floating catchment area method for measuring spatial accessibility to medical clinics in Montreal. BMC Health Serv Res 11:166.

Ni J, Liang M, Lin Y, Wu Y, Wang C, 2019. Multi-mode two-step floating catchment area (2SFCA) method to measure the potential spatial accessibility of healthcare services. ISPRS Int J Geo-Inf 8:236.

Pan J, Zhao H, Wang X, Shi X, 2016. Assessing spatial access to public and private hospitals in Sichuan, China: The influence of the private sector on the healthcare geography in China. Social Sci Med 170:35-45.

Penchansky R, Thomas JW, 1981. The concept of access: definition and relationship to consumer satisfaction. Med Care 19 2:127-40.

Shah TI, Bell S, Wilson K, 2016. Spatial accessibility to health care services: identifying under-serviced neighbourhoods in 
Canadian urban areas. PLoS One 11:e0168208.

Shen Q, 1998. Location characteristics of inner-city neighborhoods and employment accessibility of low-wage workers. Environ Plann B Plann Des 25:345-65.

Smith CM, Fry H, Anderson C, Maguire H, Hayward AC, 2017. Optimising spatial accessibility to inform rationalisation of specialist health services. Int J Health Geogr 16:15.

Song J, Tong X, Wang L, Zhao C, Prishchepov AV, 2019. Monitoring finer-scale population density in urban functional zones: a remote sensing data fusion approach. Landsc Urban Plan 190:UNSP 103580.

Stevens FR, Gaughan AE, Linard C, Tatem AJ, 2015. Disaggregating census data for population mapping using random forests with remotely-sensed and ancillary data. PLoS One 10:UNSP e0107042.

Tao Z, Cheng Y, Zheng Q, Li G, 2018a. Measuring spatial accessibility to healthcare services with constraint of administrative boundary: a case study of Yanqing District, Beijing, China. Int J Equity Health 2018;17:7.

Tao Z, Yao Z, Kong H, Duan F, Li G, 2018b. Spatial accessibility to healthcare services in Shenzhen, China: improving the multi-modal two-step floating catchment area method by estimating travel time via online map APIs. BMC Health Serv Res 18:345.

The People's Government of Hainan Province, 2018. Notice of the People's Government of Hainan Province on the issuance of action plan for standardization construction of primary healthcare institution in Hainan Province. Available from: http://www.hainan.gov.cn/hainan/szfbgtwj/201811/37479119e 1d34c03934b8c0ce54dbf67.shtml Accessed: 22 November 2019.

Voigtländer S, Deiters T, 2015. Minimum standards for the spatial accessibility of primary care: a systematic review. Gesundheitswesen 77:949-57.

Wan N, Zhan FB, Zou B, Chow E, 2012. A relative spatial access assessment approach for analyzing potential spatial access to colorectal cancer services in Texas. Appl Geogr 32:291-9.

Wang F, 2012. Measurement, optimization, and impact of health care accessibility: a methodological review. Ann Assoc Am Geogr 102:1104-12.

Wang L, Fan H, Wang Y, 2019. Fine-resolution population mapping from international space station nighttime photography and multisource social sensing data based on similarity matching. Remote Sens 11:1900.

Wang X, Pan J, 2016. Assessing the disparity in spatial access to hospital care in ethnic minority region in Sichuan Province, China. BMC Health Serv Res 16:399.

Wang X, Yang H, Duan Z, Pan J, 2018. Spatial accessibility of primary health care in China: A case study in Sichuan Province. Social Sci Med 209:14-24.

Yang J, Mao L, 2018. Understanding temporal change of spatial accessibility to healthcare: An analytic framework for local factor impacts. Health Place 51:118-24.

Yang X, Ye T, Zhao N, Chen Q, Yue W, Qi J, Zeng B, Jia P, 2019. Population mapping with multisensor remote sensing images and point-of-interest data. Remote Sens 11:574.

Ye T, Zhao N, Yang X, Ouyang Z, Liu X, Chen Q, Hu K, Yue W, Qi J, Li Z, Jia P, 2019. Improved population mapping for China using remotely sensed and points-of-interest data within a random forests model. Sci Total Environ 658:936-46.

Yip W, Fu H, Chen AT, Zhai T, Jian W, Xu R, Pan J, Hu M, Zhou Z, Chen Q, Mao W, Sun Q, Chen W, 2019. 10 years of healthcare reform in China: progress and gaps in Universal Health Coverage. Lancet 394:1192-204.

Zhang F, Li D, Ahrentzen S, Zhang J, 2019a. Assessing spatial disparities of accessibility to community-based service resources for Chinese older adults based on travel behavior: a city-wide study of Nanjing, China. Habitat Int 88:101984.

Zhang S, Song X, Wei Y, Deng W, 2019b. Spatial equity of multilevel healthcare in the metropolis of Chengdu, China: a new assessment approach. IJERPH 16:493.

Zhu L, Zhong S, Tu W, Zheng J, He S, Bao J, Huang C, 2019. Assessing spatial accessibility to medical resources at the community level in Shenzhen, China. Int J Environ Res Public Health 16:242. 